\title{
Methods of Accelerating Orthodontic Teeth Movement
}

\author{
Liuyi Chen, Ning Gao, Lei Huang, Li Gu, Zhi Wang, Jun Zeng, Lihua Li* \\ School of Medicine, North Sichuan Medical College, Fujiang Road, NanChong, China \\ *Correspondence Author, angel_li77@163.com
}

\begin{abstract}
Traditional orthodontic treatment is a long process that usually takes 2-3years.Such sophisticated orthodontic equipment as well as long term treatment duration may not only buck the cutting edge trend of "comfort, aesthetics, function and efficiency" in the orthodontic field but contribute to higher risks of complications. Thus, it is pivotal to give top priority to normal dentofacial functions, facial aesthetics as well as short treatment duration under the premise of comfort treatment. Nowadays, numerous adjunctive interventions have been developed by domestic and foreign researchers to accelerate orthodontic teeth movement, which are mainly divided into two categories: surgical intervention and non-surgical intervention. The authors of this thesis have reviewed multiple vital factors accelerating the speed of orthodontic teeth movement to provide readers with wide clinical inspirations so as to optimize the experience of the patient and reduce the incidence of orthodontic complications.
\end{abstract}

Keywords: Orthodontics, Teeth movements, Acceleration, Adjunctive intervention.

\section{Introduction}

Contemporary orthodontic treatment technology develops around the "comfort, aesthetics, function and efficiency" demand, the requirement of orthodontic effect is not limited to neatly arranged teeth, the quality of life (QoL)of the patient during the orthodontic treatment process is also vital clinical content needed to be focused on[1].Under normal circumstances, orthodontic teeth movement speed is about $1 \mathrm{~mm} / \mathrm{month}[2]$, treatment duration is generally 2-3 years, such long treatment time and complex device may lead to dental caries[3], gum disease[3], periodontitis[3], and even root absorption[4], to name but few. Frequent re-visits may reduce patient's compliance then affect the final treatment effect. Therefore, how to achieve the improvement of dental and jaw function, the aesthetic enhancement and to shorten the time of treatment under the premise of comfortable treatment is one of the important priorities of contemporary orthodontics. Based on the papers, the methods to accelerate orthodontic teeth movement are mainly divided into two categories: surgical intervention (surgical method, surgical mode) and non-surgical intervention (chemical method, physical method and biological method). The authors of this thesis summarized the relevant papers to provide readers with clinical ideas to optimize the orthodontic effect of the patient and reduce the incidence of orthodontic complications.

\section{Surgical Intervention}

\subsection{Surgical Method}

Compared with traditional orthodontic treatment, surgical methods which include osteocortictomy and distraction osteogenesis are able to shorten the course of orthodontic treatment effectively, reduce the adverse risk of root absorption and gingival atrophy[5].

\subsubsection{Osteocorticotomy}

Traditional osteocortictomy means the opening of the flap after linear incisions in the patient's lip/cheek side and tongue/palate side[6], this method is easier to accelerate the movement of orthodontic teeth by destroying the continuity of bone cortex, producing regional acceleratory phenomenon (RAP), promoting demermineralization and remineralization of local alveolar bone[7] and accelerating the alveolar bone reconstruction. However, this surgery has many adverse reactions which may not only lead to teeth inactivation and osteonecrosis, but increase the risk of periodontal injury. After nearly a hundred years of clinical practice, this method has been improved, including periodontally accelerated osteogenic orthodontics (PAOO), piezocision and microosteoperforation (MOP).

\section{(a) Periodonally assisted accelerated Orthodontics (PAOO)}

Wilcko et al.[8] proposed PAOO in 2001, a novel surgical modality that combines selective osteocortictomy with local granular bone graft on the theoretical base of RAP. Compared with traditional osteocorticotomy, PAOO is beneficial to maintain periodontal health and long-term stability of orthodontic treatment as well as increase the mass of alveolar bone[9]. However, the defects such as increasing the frequency of re-visits and the duration of operation next to the dental chair[10]. Moreover, its long-term effect on dental pulp remains to be further observed.

(b) Piezocision

In 2009, Dibart et al.[11] proposed piezocision, which requires no flap splitting but uses more "intelligent" means to accelerate orthodontic teeth movement - controlling the distance between the teeth by implants or prostheses. Such method replaces ordinary scalpel with ultrasonic bone knife in order to make the operation more precise which contributes to wound healing while also decreases the damage of periodontal tissue. However, this procedure requires high compliance with the patient. Examination of patient's orthodontic treatment is required weekly or two weeks after surgery. What's more, its long-term effect is not clear[12-13].

(c) Micro-osteoperforation (MOP) 
MOP, a minimally invasive procedure, is featured with micro perforation on the target bone cortex without flap inversion, which is easier to perform and less invasive, proposed by Alikhani et al.[14] in 2013. Its molecular mechanism of accelerating orthodontic teeth movement is still under controversy, possibly through aseprtic inflammation of MOP mediated by proinflammatory cytokines[15]. ALKEBSI et al.[16] found high satisfaction of the patient and compliance with MOP. Therefore, MOP may have more advantages than traditional one, especially for adult orthodontic patients with insufficient periodontal tissue response[17].

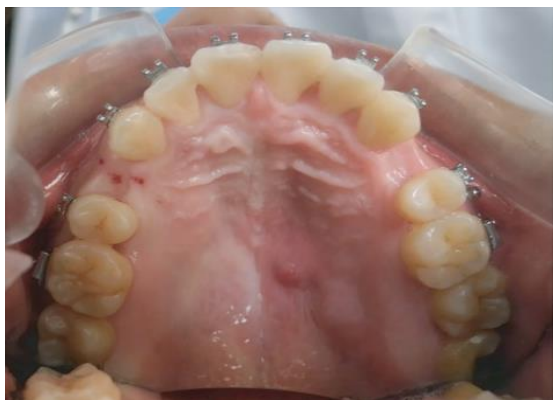

Figure 1: MOP area at first premolar extraction[15]

\subsubsection{Distraction osteogenesis}

(a) Periodontal ligament distraction (PDLD)

In 1998, Liu et al.[18] proposed PDLD, which suggested that the periodontal ligament can be regarded as a "chink" between the teeth and alveolar bone, after applying orthodontic force, the tension side of the periodontal membrane will produce new bones. In the related experimental study conducted by Ma Wensheng et al.[18-19], the expression of BMP-2 and the metabolism of type I and III collagen in the periodontal ligament of orthodontic teeth on the tensioned side are analyzed which suggests that this is to accelerate periodontal tissue remodeling by strengthening the distraction force on the tensioned side of the periodontal ligament of orthodontic teeth and stimulating new bone formation. It has also been experimentally shown that when mesial force is applied to orthodontic teeth after PDLD, the risk of distal inclination of orthodontic teeth is increased, and may even accelerate the resorption of orthodontic teeth roots[20]. Therefore, the clinical effects of PDLD require further observation.

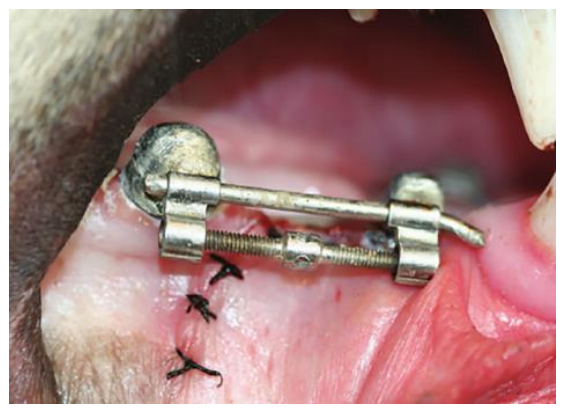

Figure 2: Image of the surgical side of canine periodontal membrane pulling osteoblastic experiment[19]

(b) Dentoalveolar Distraction (DAD)

$\mathrm{DAD}$, that is, by incising the bone cortex at the orthodontic alveolar fossa to form bone segment that can stretch and move the orthodontic root, proposed by Iseri et al. in 2005[21]. The external force applied to orthodontic teeth is transmitted to the peripheral bone of the bone segment through the periodontal ligament, which in turn causes differentiation of osteoblast and osteoclast in the periodontal tissue. The mechanism of DAD accelerating orthodontic teeth movement is mainly to stretch the two broken ends of the bone segment line in order to form new bone under intermittent distraction force then accelerate the overall movement of both the bone segment and the orthodontic teeth. Some studies have suggested that DAD can rapidly improve the patient's appearance and shorten the orthodontic treatment time[22] .

\subsection{Surgical Mode}

The SFA (Surgery-First Approach) orthodontic mode advocates orthognathic surgery followed by orthodontic treatment, so as to achieve the purpose of shortening the treatment cycle and reducing the difficulty of orthodontics[23-24]. Among them, sagittal split ramus osteotomy (SSRO), maxillary LeFort I osteotomy and horizontal osteotomy genioplasty are all common at home and abroad[25]. SFA provides a brand-new treatment concept for the treatment of the patient with dentofacial deformities[26].

In 2009, Nagasaka et al.[27] successfully used SFA for the first time, which was later used to treat malocclusion. The results of the study revealed that SFA can not only improve the patient's chief complaints at the early stage of treatment, but also accelerate the movement speed of orthodontic teeth after surgery. Liao et al.[28] compared the treatment methods of "orthodontics first" and "surgery first", the difference between the two groups was statistically significant: The total treatment duration of the "orthodontics first" group was $(512 \pm 103)$ Day, significantly longer than that of the "surgery first" group (342 \pm 127$)$ Day. Thus, the orthodontic treatment course for patients with dentofacial deformities can be shortened by preferentially performing jaw surgery. At present, the papers related to SFA are mainly cases reports as well as reviews which mean there is still a lack of a large number of animal experiments and prospective studies to confirm its clinical effect.

\section{Non-surgical Intervention}

Compared with the former, non-surgical intervention has the biggest advantage of being non-invasive or minimally invasive to the issues. Therefore, patients are generally higher acceptable to it during the orthodontic process. At present, non-surgical intervention methods for orthodontics involve chemical, physical and biological fields altogether.

\subsection{Chemical}

\subsubsection{Hormone}

Currently, scholars from home and abroad have carried out a lot of studies on the effect of hormone drugs on orthodontic teeth movement. Hormones that are able to accelerate orthodontic teeth movement including prostaglandin E2 (PGE2)[29]; Thyroid hormone (TH)[30]; 1, 25-dihydroxy vitamin D3 (1, 25-DHCC)[31]; Parathyroid hormone (PTH) [32], etc. Seif et al.[33] proved the synergistic effect of PGE2 and $\mathrm{TH}$ in accelerating orthodontic teeth movement through 
combined injection of them in rats submucosa. However, such studies are still in the laboratory stage, and the clinical effects of it need to be further evaluated. 1, 25-dhcc and PTH is capable of promoting differentiation of osteoclast and accelerate orthodontic teeth movement by regulating the expression of nuclear factor- $\mathrm{\kappa B}$ receptor-activating factor ligand (rank-1)[34-35]. In addition, estrogen acts on estrogen receptor $(E R \alpha)$ to inhibit osteoclast differentiation and stimulates the differentiation of osteoblast[36-37]. The estrogen level in women changes rhythmically as a result of the menstrual cycle: the level is low during the menstrual period when the orthodontic teeth move quickly, therefore, choosing the menstrual period as the re-visits time may effectively shorten the orthodontic duration. Since most of the experimental subjects of hormone-related studies are animals and the adverse reactions have not been clarified, the clinical effects require further studies.

\subsubsection{Traditional Chinese Medicine (TCM)}

Among the studies on the acceleration of orthodontic teeth movement by exogenous drugs, TCM has attracted the attention of scholars because of its wide range of sources. Yuan WenXiu et al.[38] found that osthole could increase the number of osteoclasts in periodontal tissues at the early stage of orthodontic in the rat experiments, thus accelerating orthodontic teeth movement. Liu Shu et al.[39] showed that salvia miltiorrhiza may effectively accelerate orthodontic teeth movement, through the mechanism of the up-regulation of VEGF expression in osteoclasts in periodontal tissues at the initial stage of orthodontic teeth loading. Lin Peng et al.[40] proved that baicalin can enhance the expression of Runx 2 in periodontal tissues of rats, promote alveolar bone remodeling and inhibit palindromia of orthodontic teeth. Zhang Lina et al.[41] reported that drynaria rhizome is effectively in promoting the proliferation and differentiation of osteoclasts in orthodontic periodontal tissues, thus speeding up orthodontic teeth movement. However, some scholars have proved that drynaria rhizome is apt to inhibit the activity of cathepsin $\mathrm{K}$ in osteoclasts of mice, thus reducing bone resorption[42]. As the specific mechanism and systemic effects of some TCM are still unclear, more studies are needed to further explore the mechanism of TCM to accelerate orthodontic teeth movement.

\subsection{Physical}

\subsubsection{Laser}

Compared with traditional surgical treatment, laser therapy has the advantage of less blood loss, so it owns a broad prospects in oral clinic. Despite many kinds of laser machines in China, there is no unified parameters and operating guidelines[43]. For a better development, its clinical usage in orthodontics needs to be standardized.

Existing studies prefer the weak laser to accelerating the orthodontic process, which can produce biological effects but will not cause irreversible tissue damage[44]. Animal experiments have shown that the weak laser irradiation may improve the expression of various related substances in periodontal tissues, thus accelerating the vascularization of them, promoting osteoclast activation, inducing the differentiation of osteoblast and accelerating orthodontic bone remodeling[45-46]. In addition, laser treatment can also alleviate orthodontic adverse reactions, including relieving pain at the initial stage of orthodontic[47], making help of treating gingival hyperplasia in orthodontic process[48], preventing peripheral inflammation of implant of orthodontic teeth[49], and relieving Dental anxiety (DA) in some orthodontic patients[50]. Therefore, laser therapy may have a positive effect on accelerating the orthodontic process, but the standard of using it have not been unified, so the clinical application has not been widely carried out.

\subsubsection{Ultrasonic}

Li Bo et al.[51] pointed out that ultrasound can promote orthodontic teeth movement by affecting periodontal reconstruction, especially low-intensity pulsed ultrasound (LIPUS). The mechanism may be that LIPUS is able to affect the expression of a variety of cytokines and promote the absorption as well as reconstruction of alveolar bone. Luan Liyang et al.[52] found that pulsed ultrasound with high intensity $\left(44 \mathrm{~W} / \mathrm{cm}^{2}, 28 \mathrm{KHz}, 10 \mathrm{~s} / \mathrm{d}\right)$ could significantly increase the number of formation of osteoclast in periodontal tissues. However, there are still few researches on the usage of ultrasound in orthodontics. The optimal intensity of it in clinical application is still controversial. Further research is needed to guide the scientific and rational application of ultrasound in orthodontic adjunctive therapy.

\subsubsection{Vibration Load}

Vibration load means that a physical vibration with appropriate frequency is applied to the orthodontic teeth and alveolar bone. Clinical tools of it include electric teethbrush and Acceledent device $(30 \mathrm{~Hz}$ low-intensity precision vibration device). The brands and vibration frequencies of electric teethbrushes used in clinical experiments are diverse. It is worth noting that the data bias for its experimental results of accelerating orthodontic teeth movement would decrease when the frequency of electric teethbrushes is $30 \mathrm{~Hz}$, with the same effect as Acceledent device[53-54]. Some studies suggest that vibration load can accelerate orthodontic teeth movement by activating the RANK/RANKL pathway and inducing signal molecules such as MAPK (MitogenActivated Protein KiNase), C-FOS as well as nitric oxide to stimulate the differentiation of osteoclast then accelerate alveolar bone remodeling[55]. However, the results of this study shows that the data of acceleration of orthodontic teeth movement by vibration load is not statistically significant. Therefore, the clinical effect of vibration load of accelerating orthodontic teeth movement requires further researches.

\subsection{Biological}

\subsubsection{Exosomes}

Exosomes are a class of extracellular vesicles (EVs) containing lipids, nucleic acids and proteins that are synthesized and secreted by various cells in the body with different functions[56]. For example, exosomes from periodontal stem cells can accelerate alveolar bone remodeling by upregulating osteogenic-related factor RUNX2 and angiogenic factor VEGFR2[57-58]. L. Shannon Holliday 
et al.[59] argued that the amount of EVs in the gingival crevicular fluid (GCF) can be used as a biomarker to indicate the patient's orthodontic teeth movement status, the degree of root resorption, as well as other periodontal conditions. Based on the easy preservation and accessibility of exosomes, Ying Zheng et al.[60] proposed that peripheral mononuclear cells can be obtained during the differentiation process of cells drawn from the patient's venous blood which the exosomes are produced. The can exosomes be used to stimulate the osteogenesis of the alveolar bone. However, the research on exosomes is still in the laboratory stage and the clinical effects need to be further confirmed.

\section{Conclusion}

It has been one of the hot topics in orthodontic researches at home and abroad that to accelerate the movement of orthodontic teeth while facial aesthetics and function are guaranteed. Existing interventions are mainly divided into surgical intervention (surgical method, surgical mode) and non-surgical intervention (chemical method, physical method and biological method). At present, scholars have conducted a large number of studies on orthodontic interventions to accelerate orthodontic teeth movement, but most of them are based on animal experiments, and are more at the morphological level, the differences in tissue structure and cellular response between humans and animals have not been comprehensively considered. Thus, the results of animal experiments can only be used as reference. In general, surgical interventions are usually more effective while they are less acceptable to patients for inevitably cause additional trauma which are the part need for improvement; On the whole, no-surgical interventions are higher acceptable by patients for the advantages of less side effects and non-invasive or minimally invasive to the issues. However, the specific irradiation duration, irradiation band and other data are not yet unified for physical methods such as laser and electromagnetic waves; Chemical methods such as hormones, herbs and biological methods such as exosomes, also need to be further explored for the specific mode of operation. In summary, an adjunctive intervention to accelerate orthodontic teeth movement that balances safety, effectiveness, and patient's satisfaction still needs to be continuously explored by a wide range of scholars.

\section{Acknowledgement}

This paper is financially supported by the Fund Project: Sichuan Provincial College Students' Innovation and Entrepreneurship (NO.: S202010634119).

\section{Statement}

All data in the paper are real and authentic

\section{References}

[1] AlSeraidi Maryam, Hansa Ismaeel, Dhaval Fadia et al. The effect of vestibular, lingual, and aligner appliances on the quality of life of adult patients during the initial stages of orthodontic treatment $[\mathrm{J}]$. Progress in Orthodontics, 22(1), pp.3-3, 2021.
[2] Zhang Nan, Tao Hong. Analysis of the application value of bag design combined with tension-free suture in accelerated periodontal osteogenesis orthodontics[J]. Chinese Aesthetic Medicine, 29(10), pp.149-152, 2020.

[3] Ren Jiajie, Du Zhen, Lin Jiang. Applied research glass ionomer cement with $\mathrm{TiO} 2$ nanoppapers in orthodontic treatment[J]. Journal of Nanoscience and Nanotechnology, 21(2), pp. 1032-1041, 2021.

[4] Hatrom Abdulkarim A., Howait Mohammed S., Zawawi Khalid H., et al. Pulp volume changes after piezocisionassisted teeth movement: a randomized clinical trial[J]. BMC Oral Health, 21(1), pp.28-28, 2021.

[5] Chen Liqiong, Zhang Xiaorong. Research progress of orthodontic treatment assisted by cortical osteotomy[J]. Chinese Journal of Practical Stomatology, 13(06), pp.369-374, 2020.

[6] Chen Liqiong, Zhang Xiaorong. Research progress on the biological mechanism of accelerated teeth movement in orthodontic treatment with cortical osteotomy[J]. Shandong Medicine, 60(25), pp.103-106, 2020.

[7] Wilcko W, Wilcko MT. Accelerating teeth movement: the case for corticotomy-induced orthodontics[J]. Am J Orthod Dentofacial Orthop, 144 (1), pp.4-12, 2013.

[8] Wilcko WM, Wilcko T, Bouquot JE, et al. Rapid orthodontics with alveolar reshaping: Two case reports of decrowding[J]. Int JP Eriodontics Restorative Dent, 21(1), pp.9-19, 2001.

[9] Li Hanyue, Xia Lulu, Hua Xianming. Progress in clinical application of accelerated periodontal osteogenesis orthodontics[J]. International Journal of Stomatology, 47(02), pp.206-211, 2020.

[10] Yang Yuhong, Tang Qian. Orthodontic treatment with periodontal assisted accelerated osteogenesis[J]. Chinese Journal of Stomatological Research (Electronic Edition), 11(01), pp.49-52, 2017.

[11] Dibart S, Sebaoun JD, Surmenian J. Piezocision: A minimally invasive, periodontally accelerated orthodontic teeth movement procedure $[\mathrm{J}]$. Compend Contin Educ Dent, 30(6), pp.342-344, 346, 348-350, 2009.

[12] Hanife Nuray Yilmaz, Evin Alakus, Buket Erdem, et al. Effect of piezocision on molar intrusion in open-bite treatment using a modified MEAW technique[J]. Journal of Orofacial Orthopedics, pp.1-12, 2020.

[13] Da Haiqin, Han Shuang. Clinical application of Piezocision accelerated orthodontic treatment for mandibular first molar gap closure[J]. Journal of Practical Stomatology, 35(01), pp.77-80, 2019.

[14] Alikhani M, Raptis M, Zoldan B, et al. Effect of microosteoperforations on the rate of teeth movement $[\mathrm{J}]$. Am J Orthod Dento Facial Orthop, 144(5), pp.639-648, 2013.

[15] Liu Zhifeng, Yang Ruihua, Sun Jinlong, et al. Clinical study of microperforation of alveolar bone for accelerated teeth movement in adult orthodontics[J]. Journal of Translational Medicine, 9(02), pp.119-121, 2020.

[16] Alkebsi A, AL Maaitah E, AL Shorman H, et al. Three-dimensional assessment of the effect of microosteoperforation $\mathrm{s}$ on the rate of teeth movement during canine retraction in adults with Class II malocclusion: A randomized controlled clinical trial[J]. Am J Orthod Dentofacial Orthop, 153(6), pp.771-785, 2018. 
[17] Liu Zhifeng, Yang Ruihua, Sun Jinlong, et al. Clinical study of micro perforation of alveolar bone for accelerated teeth movement in adult orthodontics[J]. Journal of Translational Medicine, 9(02), pp.119-121, 2020.

[18] Ma Wensheng. Experimental and application study of rapid orthodontic teeth movement with distraction osteogenesis[D]. Hebei Medical University, 2009.

[19] Feng Lixiao. Effect of periodontal membrane distraction osteogenesis on the metabolism of type I and III collagen in canine periodontal membrane[D]. Hebei Medical University, 2008.

[20] Liu Lili. Study on the expression of RANKL and OPG in periodontal tissue of canine periodontal membrane distraction with rapid teeth pressure[D]. Hebei Medical University, 2010.

[21] Kateel SK, Agarwal A, Kharae G, et al. A comparative study of canine retraction by distraction of the periodontal ligament and dentoalveolar distraction methods[J]. J Maxillofac Oral Surg. 15(2), pp.144-55, 2016.

[22] Uzuner F Deniz, Darendeliler Nilufer. Dentoalveolar surgery techniques combined with orthodontic treatment: A literature review[J]. European Journal of Dentistry, 7(2), pp.257-265, 2013.

[23] Sun Yuchen, Ge Baozhen, Zhang Yimo. A review of 3D object information measurement technology[J]. Optoelectronics Laser, pp.248-254, 2004(02).

[24] Li K, Li X L, Lei K, et al. Comparison of the effect of "operation first" mode and orthodontic-orthodontic combined mode in the treatment of adult skeletal malocclusion III $[\mathrm{J}]$. Hainan medical, 28(19), pp.3156-3159, 2017.

[25] Wei Yao. Application of surgical priority in orthodontic treatment of adult bone class III malocclusion[D]. Dalian Medical University, 2014.

[26] Liu Jiaqi, Cao Zhiwei, Bi Ruiye. Application of surgical advance mode in the treatment of odontomaxillofacial deformity[J], 27(11), pp.733-738, 2019.

[27] Nagasaka H, Sugawara J, Kawamura H, et al. "Surgery first" skeletal class III correction using the Skeletal Anchorage System[J]. J Clin Orthod, 43(2), pp.97-105, 2009.

[28] Liao YF, Chiu YT, Huang CS, et al. Presurgical orthodontics versus no presurgical orthodontics: treatment outcome of surgical-orthodontic correction for skeletal class III open bite[J]. Plast Reconstr Surg, 126(6), pp.2074-2083, 2010.

[29] Cağlaroğlu M, Erdem A. Histopathologic investigation of the effects of prostaglandin E2 administered by different methods on teeth movement and bone metabolism[J]. Korean J Orthod, 42(3), pp.118-28, 2012.

[30] Kacprzak Alicja, Strzecki Adrian. Methods of accelerating orthodontic teeth movement: A review of contemporary literature[J]. Dental and Medical Problems, 55(2), pp.197-206, 2018.

[31] Almoammar K. Vitamin D and orthodontics: An insight review[J]. Clin Cosmet Investig Dent, pp.165-170, 2018.

[32] Yao Li, Xiao-Yan Chen, ZhengLong Tang, et al. Differences in accelerated teeth movement promoted by recombinant human parathyroid hormone after mandibular ramus osteotomy[J]. American Journal of Orthodontics and Dentofacial Orthopedics, 155(5), pp.670-680, 2019.

[33] Seifi Massoud, Hamedi Roya, Khavandegar Zohre. The effect of thyroid hormone, prostaglandin e2, and calcium gluconate on orthodontic teeth movement and root resorption in rats[J]. Journal of Dentistry, 16(1), pp.35-42, 2015.

[34] Zarei Allahdad, Morovat Alireza, Javaid Kassim, et al. Vitamin D receptor expression in human bone tissue and dose-dependent activation in resorbing osteoclasts[J]. Bone Research, 4, pp.16030, 2016.

[35] Lu Wenxin, Li Xue, Yang Yan, et al. PTH/PTHrP in controlled release hydrogel enhances orthodontic teeth movement by regulating periodontal bone remodaling $[\mathrm{J}]$. Journal of Periodontal Research, 56(5), pp.885-896, 2021.

[36] Lanzhi Deng, Yongwen Guo. Estrogen effects on orthodontic teeth movement and orthodontically induced root resorption $[\mathrm{J}]$. Archives of Oral Biology, 118, pp.104840-104840, 2020.

[37] Wang Bin, Yang Xi, Zhou Jianping, et al. Effect of Afterloading on the movement of orthodontic teeth in adolescent women[J]. Chinese Journal of Tissue Engineering Research, 18(15), pp.2332-2337, 2014.

[38] Yuan Wenxiu, Wang Xuxia, Zhang Lina, et al. Effect of osthole on periodontal tissue remodeling in orthodontic rats[J]. Shanghai Journal of Stomatological Medicine, 28(06), pp.561-566, 2019.

[39] Liu Shu, Zheng Zhijun, Zuo Changyan, et al. Effect of salvia miltiorrhiza on expression of vascular endothelial growth factor in periodontal tissue of orthodontic rats $[\mathrm{J}]$. Electronic Journal of General Stomatology, 5(36), pp.3-5, 2018.

[40] Lin Peng, Wei Zhuliang, Wang Yiling, et al. Effect of baicalin on Runx2 expression during orthodontic retention in rats[J]. Journal of Clinical Stomatology, 35(02), pp.75-78, 2019.

[41] Zhang Lina, Wang Xuxia, Zhang Wenjuan, et al. Effect of bone crusher hydrating decoction on osteoclast during orthodontic teeth movement in rats[J]. Shanghai Journal of Traditional Chinese Medicine, 45(06), pp.72-75, 2011.

[42] Song Jia, ZHAO Gang, Song Chunlei. Effect of bone subufu on the expression of RANKL in periodontitis orthodontic rats[J]. Medical Information, 32(04), pp.85-87, 2019.

[43] Liu Zizhu, Xu Yuhong. Research progress of semiconductor laser in orthodontic treatment[J]. Medical Review, 26(09), pp.1765-1769, 2020.

[44] Jiang C, Li Z, Quan H, et al. Osteoimmunology in orthodontic teeth movement[J]. Oral Dis, 21(6), pp.694-704, 2015.

[45] Ma Rui. Effect of low intensity laser combined with bone microperforation on teeth movement in orthodontic rats[D]. Zunyi Medical University, 2020.

[46] Yuan Ziyi, Liu Jun, Yi Jicui, et al. Effects of low energy laser irradiation on the expression of P-ErK $1 / 2$ in periodontal membrane cells of orthodontic rats $[\mathrm{J}]$. Journal of Medical College of Qingdao University, 52(03), pp.315-318, 2016.

[47] Qiu Ni, Luo Xinxiao, Li Nan, et al. Clinical evaluation of low intensity laser therapy for pain relief during 
orthodontic treatment[J]. Journal of Practical Stomatological Medicine, 34(06), pp. 830-833, 2018.

[48] Bai Qing, Li Tao, Shang Xiaopan, et al. Comparison of clinical efficacy of Nd: YAG laser, Er: YAG laser and traditional gingival resection for orthodontic gingival hyperplasia[J]. Using Lasers, 38(02), pp.310-314, 2018.

[49] Li Yan, Duan Xiaoyan. Clinical study of semiconductor laser irradiation for prevention of orthodontic microimplantitis[J]. Laser Journal, 37(04), pp.131-134, 2016.

[50] Wang Yangyang, Zhao Chanyuan, Chen Xibo, et al. Effects of emotion therapy and Er laser therapy on dental anxiety in adolescents with orthodontic fenestration[J]. Hebei Medical, 25(07), pp.1135-1140, 2019.

[51] Li Bo, Zhao Zhihe. A study on the effect of orthodontic teeth movement on dental performance $[\mathrm{J}]$. Chinese Journal of Stomatology, 37(06), pp.648-655, 2019.

[52] Luan Liyang, Jiang Jiuhui, Li Cuiying, et al. Experimental study of ultrasonic accelerated orthodontic teeth movement[J]. Journal of Stomatological Medicine, 32(02), pp.109-112, 2016.

[53] Keerthana P, Diddige R, Chitra P. Performance comparison of vibration devices on orthodontic teeth movement - A systematic review and meta-analysis[J]. J Oral Biol Craniofac Res, 10(4), pp.814-823, 2020.

[54] Dubravko Pavlin, Ravikumar Anthony, Vishnu Raj, et al. Cyclic loading (vibration) accelerates teeth movement in orthodontic patients: A double-blind, randomized controlled trial[J]. Seminars in Orthodontics, 21(3), pp.187-194, 2015.

[55] Muhammad Azeem, Ambreen Afzal, Saqib Ali Jawa, et al. Effectiveness of electric teethbrush as vibration method on orthodontic teeth movement: a split-mouth study[J]. Dental Press Journal of Orthodontics, 24(2), pp.49-55, 2019.

[56] Liu Shibo, Liu Xian. Research progress of exosomes from different sources in bone defect repair[J]. West China Journal of Stomatological Medicine, 38(02), pp.193-197, 2020.

[57] Gong Shengkai, Sun Zaiang, Wang Xiao, et al. Research progress of exosomes derived from odontogenic stem cells[J]. Journal of Stomatological Medicine, 40(12), pp.1147-1151, 2020.

[58] Gong Yujia, Liu Yi. New progress in functional study of exosomes derived from odontogenic stem cells[J]. Chinese Journal of Practical Stomatology, 13(11), pp.651-654, 2020.

[59] Holliday L Shannon, Truzman Estella, Zuo Jian, et al. Extracellular vesicle identification in teeth movement models[J]. Orthodontics \& Craniofacial Research, 22(1), pp.101-106, 2019.

[60] Zheng Ying, Liu Yi. Function of exosome in bone remodeling and its role in orthodontic teeth movement[J]. Chinese Journal of Practical Stomatology, 13(11), pp.646-650, 2020. 